\title{
Semantically Homogeneous Segmentation with Nonparametric Region Competition*
}

\author{
Ming TANG ${ }^{\dagger}$ Jing XIAO and SongDe MA \\ National Laboratory of Pattern Recognition, P.O.Box 2728, Beijing 100080, P.R. China
}

\begin{abstract}
This paper presents a nonparametric region competition algorithm which combines scale-space clustering and region competition to segment the image. It also proposes a formal and general procedure to automatically find the initial regions. Our algorithm can also segment an image into regions which are not homogeneous in the sense of statistics, but is homogeneous in the sense of semantics with respect to the segmentation context.
\end{abstract}

Keywords Segmentation, region growing, region competition

\section{Introduction}

Thresholding techniques [5] and scale-space clustering [4] often generate irregular boundaries and undesired small holes. And the discrimination points in the histogram may not be optimal in the sense of minimizing the classification error. Therefore, other criterion has to be introduced to reduce the error.

Hybrid techniques [1] [3] generally may result in better segmentation. Parametric region competition algorithm [6] is such an example. It combines attractive aspects of snakes/balloons and region growing to produce a segmentation with regular boundaries and without small holes. However, the forms of underlying density functions of the feature values have to be assumed in advance, and such assumptions are usually suspicious.

Up till now, there have been no formal and general procedure, to our knowledge, that can select the seeds for region growing and competition.

To overcome these drawbacks and to improve the segmentation of thresholding techniques and scale-space clustering, a nonparametric region competition algorithm is proposed in this paper. Nonparametric region competition al-

${ }^{*}$ This work is partially supported by Beijing Institute of Environment Feature. The first author would also thank Prof. GongQing Zhang for his help in preparing the text.

†Email: tangm@nlpr.ia.ac.cn gorithm utilizes a scale-space based $\mathrm{n}$-dimensional feature histogram ( $n \geq 1$ ) to automatically obtain the initial regions, and then adopts the strategy of region competition to determine the final regions. Our method uses a general and formal strategy to automatically obtain the initial segmentation, and can segment an image into regions which are homogeneous in the sense of semantics with respect to the segmentation context.

The rest of this paper is organized as follows. Section 2 describes the basic theory of nonparametric region competition. The method to automatically determine the seeds in our algorithm is presented in Section 3. Section 4 describes the method to approximately evaluate the conditional probabilities related to our algorithm. In section 5, the nonparametric region competition algorithm is presented. Experimental results on real images is included in Section 6. Section 7 is the conclusion.

\section{Basic Theory}

Suppose the domain $R$ of image $I$ is initially segmented into $l$ regions $R_{i}, i=1,2, \ldots, l . R=\bigcup_{i=1}^{l} R_{i}, R_{i} \cap R_{j}=$ $\Phi$ if $i \neq j$. Define the direction of $\Gamma_{i}=\partial R_{i}$ to be counter-clockwise. In the case of holes, it is clockwise. $\Gamma=\bigcup_{i=1}^{l} \Gamma_{i}$.

In order to smooth the irregular boundaries and make the resulting segmentation homogeneous individually, we refer to the basic idea of [6] and propose the following functional

$$
E[\Gamma]=\sum_{i=1}^{l}\left\{\mu \oint_{R_{i}} d s-\log P\left(\left\{\vec{x}(p) \mid p \in R_{i}\right\}\right)\right\}
$$

where $p$ is the pixel, $\vec{x}(p)$ is the feature vector of $p, P$ is probability. The first term within the braces is the length of the boundary curve $\Gamma_{i}$ for $R_{i}, \mu$ is a weight constant. The second term is related to the individual semantical homogeneity of the segmentation. The more individually homogeneous the segmentation, the smaller the second term.

Assuming the probabilities of $\vec{x}(p)$ 's to be independent, 
we have

$$
\log P\left(\left\{\vec{x}(p) \mid p \in R_{i}\right\}\right)=\int_{V_{i}} \log \hat{p}\left(\vec{x}(p) \mid p \in R_{i}\right)
$$

where $V_{i}=\left\{\vec{x}(p) \mid p \in R_{i}\right\}$.

In order to obtain the desirable segmentation, $R_{i}$ 's, i.e., $\Gamma_{i}$ 's, may deform based on the individual semantical homogeneity. Steepest descent with respect to $\Gamma$ is adopted. For any $p \in \Gamma, d p / d t=-\delta E[\Gamma] / \delta p$. Taking the functional variation (see Appendix in [6]) yields the following motion equation of $p$

$$
\frac{d p}{d t}=\sum_{i \in Q(p)}\left\{-\mu \kappa_{i(p)}+\log \hat{p}\left(\vec{x}(p) \mid p \in R_{i}\right)\right\} \overrightarrow{\mathbf{n}}_{i(p)}
$$

where $Q(p)=\left\{i \mid p \in \Gamma_{i}\right\}$, that is, the summation is done over $R_{i}$ 's for which $p$ is on $\Gamma_{i} . \kappa_{i(p)}$ is the curvature of $\Gamma_{i}$ at point $p$ and $\overrightarrow{\mathbf{n}}_{i(p)}$ is the unit normal to $\Gamma_{i}$ at point $p . \overrightarrow{\mathbf{n}}_{i(p)}$ 's point rightward along the direction of $\Gamma_{i}$.

When $\forall p \in \Gamma, d p / d t=0$, the segmentation is finished.

Before we can use Equation (2) to segment an image, we have to solve two problems, i.e., how to determine the initial segmentation, and how to calculate conditional probability $\hat{p}\left(\vec{x}(p) \mid p \in R_{i}\right)$ approximately. The following two sections will deal with them.

\section{Determining Initial Segmentation}

To determine the initial regions we first classify the image points. The following algorithm is for this purpose.

Algorithm 1 (scale-space based classification)

Step 1. Select a set of proper features to construct a $n$ dimensional feature histogram (feature space), $\hat{p}_{\nu}(\vec{x})$, of image $I$, where $n \geq 1$.

Step 2. If $\hat{p}_{\nu}(\vec{x})=0$, label $\vec{x}$ with 'NULL'.

Step 3. Compute scale space of $\hat{p}_{\nu}(\vec{x})$

$$
\hat{p}_{\sigma}(\vec{x})=\hat{p}_{\nu}(\vec{x}) * G_{\sigma}(\vec{x})
$$

where $G_{\sigma}(\vec{x})$ is Gaussian. Starting from $\sigma_{0}$, evolve $\hat{p}_{\sigma}(\vec{x})$ with $\Delta \sigma$, the step length of $\sigma$. Let $\pi(\sigma)$ be the number of peaks of $\hat{p}_{\sigma}(\vec{x})$. After $\sigma \geq \sigma_{1}, a$ valid estimation of the density function, $\hat{p}_{\sigma_{1}}(\overrightarrow{\vec{x}})$, is obtained if $\pi\left(\sigma_{1}\right)=\pi(\sigma)=\pi\left(\sigma_{2}\right)$, i.e., $\pi(\sigma)$ is stable over a range of $\sigma_{1}$ to $\sigma_{2}$, where $\sigma \in\left[\sigma_{1}, \sigma_{2}\right]$ and $\left|\sigma_{1}-\sigma_{2}\right|>v_{t}, v_{t}$ is a threshold, and there does not exist any such stable interval if $\sigma<\sigma_{1}$.

Step 4. Find $M$, the set of all peaks of $\hat{p}_{\sigma_{1}}(\vec{x})$. Different peaks define different clusters (patches).
Step 5. If feature $\vec{x}$ can reach peak $p_{m}$ with gradient increasing approach, $\vec{x}$ is assigned to the cluster defined by $p_{m}$. Determine the cluster to which each feature point belongs.

Step 6. Depending on the context of segmentation, select some classic algorithm of classification(such as c-means algorithm, etc.), and distance measurement(e.g., Euclidean measurement) to partition $M$ into $k$ classes, where $k$ is a parameter representing the required number of resulting regions and $k=0$ means $|M|$ classes are required, i.e., $k=|M|$. Correspondingly $|M|$ clusters, $\omega_{i}(i=1,2, \ldots,|M|)$, are partitioned into $k$ classes, $c_{j}(j=1,2, \ldots, k)$, too.

Step 7. For $\forall \vec{x}$, if $\vec{x}$ belongs to $\omega_{i}$, and if $\omega_{i}$ is classified into $c_{j}$ in Step 6, then classify $\vec{x}$ into $c_{j}$.

Step 8. For $\forall p \in I, I(p)=j$ if $\vec{x}(p)$ is classified into $c_{j}$.

In the above algorithm, each $\omega_{i}$ corresponds to a statistically homogeneous patch, each $c_{j}$ corresponds to a semantically homogeneous region.

In the rest of this paper, we suppose $c_{j}(j=1 ; 2, \ldots, k)$ is the $j$-th obtained class. $\omega_{i j} \subseteq c_{j}\left(i=1,2, . ., k_{j}\right)$ is the $i$-th cluster in the $j$-th class. $k$ is the number of classes. $k_{j}$ is the number of clusters in the $j$-th class. Only those feature points which are not labelled with 'NULL' are considered.

The following algorithm determines which pixels will be taken as seeds. The initial regions consist of all seeds and their background.

Algorithm 2 (determine the initial regions)

Step 1. Obtain the classification of all feature points with Algorithm 1.

Step 2. For any $\omega_{i j}$

$$
\hat{p}_{i j}\left(\vec{x} \in \omega_{i j}\right)=\frac{\hat{p}_{\sigma_{1}}(\vec{x})}{\sum_{\vec{z} \in \omega_{i j}} \hat{p}_{\sigma_{1}}(\vec{z})}
$$

For $\forall p \in I$, if $\hat{p}_{i j}(\vec{x}(p))>P_{i j}$, take $n_{w} \times n_{w}$ window centered at $p$ as a seed of the $j$-th segment, where $k \neq$ $j, 0<P_{i j}<1$ is a prescribed constant.

Step 3. Merge neighboring and overlapping seeds of the same segment to constitute a seed group.

Step 4. Remove the seed groups which are smaller than $n_{s} \times n_{s}$ and do not neighbor on the background. Background is the image areas not occupied by any seed. The remaining groups and their background constitute the initial regions

In our experiments, $n_{w}=5$ and $n_{s}=3$. It is clear that a seed group may not be homogeneous in the sense of statistics. Several statistically homogeneous seed subgroups may constitute a statistically heterogeneous seed group. 


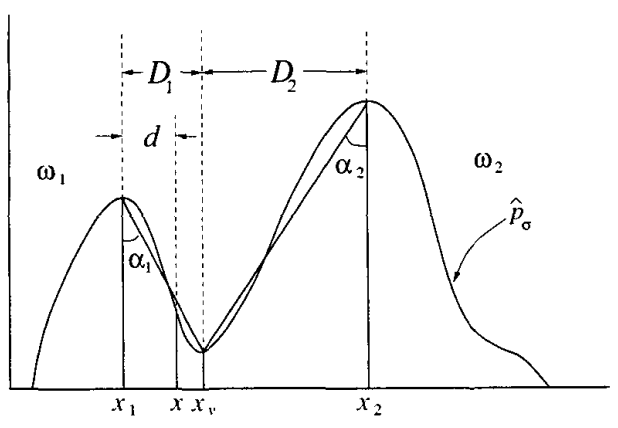

\section{Figure 1. The principle adopted in the calcu- lation of conditional probabilities. See text.}

\section{Calculating Conditional Probabilities}

The principle we employ to approximate $\hat{p}\left(\vec{x} \in \omega_{i j}\right)$ 's is illustrated in Fig.l. $\omega_{1}$ and $\omega_{2}$ represent two clusters, and belong to different classes. $x_{v}$ is their boundary. The peaks of $\omega_{1}$ and $\omega_{2}$ are $x_{1}$ and $x_{2}$, respectively. $x \in \omega_{1}$. Suppose the overlapping between $\omega_{1}$ and $\omega_{2}$ is relatively small. Now the problem is how to estimate $\hat{p}\left(x \in \omega_{1}\right)$ and $\hat{p}\left(x \in \omega_{2}\right)$. It is reasonable to assume that $\hat{p}\left(x \in \omega_{i}\right)$ depends on $\alpha_{i}$ and $\left|x-x_{i}\right|$. For the fixed $\left|x-x_{i}\right|$, the larger $\alpha_{i}$, the larger the variance of $\omega_{i}$, therefore the larger $\hat{p}\left(x \in \omega_{i}\right)$. For the fixed $\alpha_{i}$, the larger $\left|x-x_{i}\right|$, the smaller $\hat{p}\left(x \in \omega_{i}\right)$. Therefore $\alpha_{i}$ and $\left|x-x_{i}\right|$ are two proper measures for $\hat{p}\left(x \in \omega_{i}\right)$, where $i=1,2$. In addition, the larger $D_{2}$, the larger the variance of $\omega_{2}$, therefore the larger $\hat{p}\left(x \in \omega_{2}\right)$. Furthermore, it is reasonable to assume that $\hat{p}\left(x \in \omega_{1}\right)<\hat{p}\left(x \in \omega_{2}\right)$ in the case of $x \in \omega_{1}$ can only occur when $x$ is near to $x_{v}$. Therefore $\hat{p}\left(x \in \omega_{2}\right)$ should be a monotonically decreasing function of $d / D_{1}$. Based on the above consideration, we propose a method to approximately calculate $\hat{p}\left(\vec{x} \in \omega_{i j}\right)$.

Suppose that $0<\epsilon \ll 1$, that $|\bullet|$ is the number of the elements in set $\bullet$, that $B$ is the background (the image areas not occupied by any seed), that $\partial \omega_{i j}$ is the boundary of $\omega_{i j}$, and that $\vec{x}_{i j}^{(m)}$ is the peak of $\omega_{i j}$. If $\exists p \in \omega_{i t}, \exists q \in \omega_{j s}$, where $t \neq s$, such that $p$ neighbors on $q$, we call $\omega_{i t}$ and $\omega_{j s}$ neighbor on each other. After running Algorithm 1, for any $\omega_{i t}$, it is easy to find all $\omega_{j s}$ 's which neighbors on $\omega_{i t}$, where $t \neq s$.

Suppose $p \in I, p \notin B$, and $\vec{x}=\vec{x}(p) \in \omega_{h t}$. We consider the case that there exist $m$ clusters, $\omega_{j s}, s \neq t$, which neighbor on $\omega_{h t}$. All $\omega_{j s}$ 's and $\omega_{h t}$ constitute a set $\Omega_{h t}$.

Firstly, we suppose $m \geq 1$.

For $\forall \omega_{i j} \in \Omega_{h t}$, we set

$$
d_{i j}(\vec{x})=\left\|\vec{x}-\vec{x}_{i j}^{(m)}\right\|^{2},
$$

$$
\begin{gathered}
\vec{x}_{i j}=\arg \min _{\vec{y} \in \partial \omega_{i j}}\|\vec{x}-\vec{y}\|, \\
\Gamma_{i j}(\vec{x})=\left\{\vec{z} \mid \vec{z} \in \partial \omega_{i j},\left\|\vec{z}-\vec{x}_{i j}\right\|<d\right\}
\end{gathered}
$$

where $d$ is a constant, and

$$
\alpha_{i j}(\vec{x})=\frac{\sum_{\vec{z} \in \Gamma_{i j}(\vec{x})} \arccos \left(-\frac{\vec{v}(\vec{z}) \cdot(-\vec{h})}{\|\vec{v}(\vec{z})\|}\right)}{\left|\Gamma_{i j}(\vec{x})\right|}
$$

where $\vec{v}(\vec{z})$ is a vector from $\left(\vec{x}_{i j}^{(m)}, \hat{p}_{\sigma}\left(\vec{x}_{i j}^{(m)}\right)\right)$ to $\left(\vec{z}, \hat{p}_{\sigma}(\vec{z})\right)$, $\vec{h}$ is the unit vector along the histogram axis. $\left|\Gamma_{i j}(\vec{x})\right|$ is the number of the elements in $\Gamma_{i j}(\vec{x})$.

Under the postulate that the overlapping among the $\omega_{i j}$ 's is relatively small, it is reasonable to assume that the larger $\alpha_{i j}(\vec{x})$, the larger $\hat{p}\left(\vec{x} \in \omega_{i j}\right)$; and the larger $d_{i j}(\vec{x})$, the small $p\left(\vec{x} \in \omega_{i j}\right)$. Therefore, for the fixed $d_{i j}(\vec{x}), \hat{p}(\vec{x} \in$ $\omega_{i j}$ ) should be the monotonically increasing function of $\alpha_{i j}(\vec{x})$; and for the fixed $\alpha_{i j}(\vec{x}), \hat{p}\left(\vec{x} \in \omega_{i j}\right)$ should be the monotonically decreasing function of $d_{i j}(\vec{x})$.

On the other hand, we define

$$
D_{i j}(\vec{x})=\frac{\sum_{\vec{z} \in \Gamma_{i j}(\vec{x})}\left\|\vec{z}-\vec{x}_{i j}^{(m)}\right\|}{\left|\Gamma_{i j}(\vec{x})\right|}
$$

It is reasonable to assume that the larger $\left(D_{h t} / d_{h t}\right)^{2} / D_{i j}$, the smaller $\hat{p}\left(\vec{x} \in \omega_{i j}\right)$, where $i \neq h, j \neq t$. Therefore, $\hat{p}\left(\vec{x} \in \omega_{i j}\right)$ should be the monotonically decreasing function of $\left(D_{h t} / d_{h t}\right)^{2} / D_{i j}$, where $i \neq h, j \neq t$.

Synthesizing the above consideration, we define

$$
w_{i j}(\vec{x})=\left(c \cdot \sin \left(\alpha_{i j}(\vec{x})\right)+\exp \left\{-\frac{d_{i j}(\vec{x})}{s_{1}^{2}}\right\}\right) \cdot f_{i j}(\vec{x})
$$

where

$f_{i j}(\vec{x})= \begin{cases}1, & \text { if } i=h, j=t ; \\ \exp \left\{-\frac{\left(\frac{D_{h t}}{d_{h t}}-1\right)^{2} \cdot \frac{1}{D_{i j}}}{s_{2}^{2}}\right\}, & \text { otherwise. }\end{cases}$

$c \geq 0, c, s_{1}$ and $s_{2}$ are three constants.

If $m=0, w_{h t}(\vec{x})=1$.

For $\forall \omega_{u v} \in \Omega-\Omega_{h t}$, we set

$$
w_{i j}(\vec{x})=\epsilon
$$

where $\Omega$ is the set of all clusters.

Consequently, we define

$$
\hat{p}\left(\vec{x} \in \omega_{i j}\right)=\frac{w_{i j}(\vec{x})}{\sum_{w_{p q} \in \Omega} w_{p q}}
$$


where $w_{i j} \in \Omega$.

Therefore

$$
\hat{p}\left(\vec{x}(p) \mid p \in R_{j}\right)=\sum_{\omega_{i j} \in c_{j}} \hat{p}\left(\vec{x} \in \omega_{i j}\right)
$$

where $c_{j}$ is the class to which region $R_{j}$ corresponds.

In addition, we define

$$
\hat{p}(\vec{x}(p) \mid p \in B)=\left\{\min _{\omega_{i j} \in \Omega} \hat{p}\left(\vec{x} \in \omega_{i j}\right)\right\}^{2}
$$

\section{Nonparametric Region Competition}

Algorithm 3 (nonparametric region competition)

Step 1. Obtain the seeds with Algorithm 2.

Suppose the initial regions are $R_{i}^{(0)}$, where $i=$ $1,2, . ., l$.

Step 2. Based on Equation (1), move the boundaries of all regions to make them grow and compete with each other. Execute this step repeatedly until all boundaries are stable.

Obviously, the resulting regions with our algorithm can not only be individually statistically homogeneous, but also be individually semantically homogeneous in the segmentation context. We call such segmentation semantically homogeneous. However, high-level instructions are necessary.

\section{Experimental Results}

The experiment given here is about the segmentation of a color image of hand. What is shown in Fig.2(1) is its graylevel version. In this experiment, we segment the image in two ways: statistically homogeneous (Fig.2(2)), semantically homogeneous (Fig.2(3)). To obtain such two segmentations, the intensity, hue and saturation are employed. The edges of the final stable regions are shown in Fig.2(2) and (3), respectively.

\section{Conclusions}

In this paper, we presented a new segmentation scheme of nonparametric region competition, which can automatically determine the initial segmentation and segment an image into semantically homogeneous regions. Such segmentation may simplify the following procedures, such as object recognition.

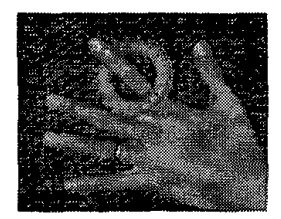

(1)

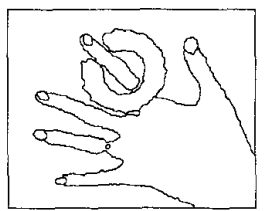

(2)

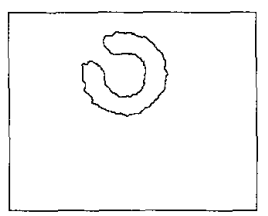

(3)

\section{Figure 2. The segmentation of a hand image. See text.}

\section{References}

[1] Besl, P.J. and Jain, R.C., Segmentation through Variable-Order Surface Fitting, IEEE T-PAMI, Vol.10, pp167-192, 1988.

[2] Haralick, R.M. and Shapiro, L.M., Computer and Robot Vision, Vol.1, Addison Wesley, Reading, MA, 1993.

[3] Pavlidis, T. and Liow, Y-T., Integrating Region Growing and Edge Detection, IEEE T-PAMI, Vol.12, No.3, pp225-233, 1990 .

[4] Roberts, S.J., Parametric and Non-Parametric Unsupervised Cluster Analysis, Pattern Recognition, Vol.30, No.2, pp261-272, 1997.

[5] Sahoo, P.K., Soltani, S. and Wong, A.K.C., A Survey of Thresholding Techniques, CVGIP, Vol.41, pp233$260,1988$.

[6] Zhu, S.C. and Yuille, A.L., Region Competition: Unifying Snakes, Region Growing and Bayes/MDL for Multiband Image Segmentation, IEEE T-PAMI, Vol.18, No.9, pp884-900, 1996. 